Supplement of

\title{
A high-resolution regional emission inventory of atmospheric mercury and its comparison with multi-scale inventories: a case study of Jiangsu, China
}

Hui Zhong et al.

Correspondence to: Yu Zhao (yuzhao@nju.edu.cn)

The copyright of individual parts of the supplement might differ from the CC-BY 3.0 licence. 


\section{SUPPLEMENT}

\section{Figure List}

Figure S1. Location of Jiangsu Province and spatial distribution of major point sources (coal consumption>90 000 metric tons/yr for CPP and OIB).

Figure S2. Bootstrap simulation analysis for Hg content in limestone, expressed as the probability bands and probability distribution function (PDF).

Figure S3. Differences in gridded emissions of $\mathrm{Hg}^{0}$ (a-c), $\mathrm{Hg}^{2+}$ (d-f), and $\mathrm{Hg}^{\mathrm{p}}$ (h-j) in Jiangsu 2010 between provincial and other inventories. From left to right column: emissions in provincial inventory minus those in NJU, THU and AMAP/UNEP. The locations of point sources with relatively large $\mathrm{Hg}$ emissions estimated in provincial inventory are indicated in the panels as well.

\section{Table List}

Table S1. Summary of calculation methods of $\mathrm{Hg}$ emissions for coal-fired power plants (CPP), cement production (CEM), iron \& steel plants (ISP) and other industrial boilers (OIB) in multi-scale inventories.

Table S2. Database of $\mathrm{Hg}$ removal efficiencies by air pollutant control device (APCD).

Table S3. Database of mass fractions of speciated Hg by APCD.

Table S4. Emission factors and Hg speciation profiles for ISP and other selected sources in Categories 2 and 3.

Table S5. Removal efficiencies and application ratios of various APCDs between multi-scale $\mathrm{Hg}$ emission inventories.

Table S6. Uncertainties of Hg emission factors for main sources, expressed as the probability distribution functions (PDF).

Table S7. Uncertainties of mass fractions of Hg speciation for main source categories, expressed as the probability distribution functions (PDF).

Table S8. The parameters contributing most to emission uncertainties by sector for 2010. The percentages indicate the contributions of the parameters to the variance of corresponding emission estimates. 
Figure S1

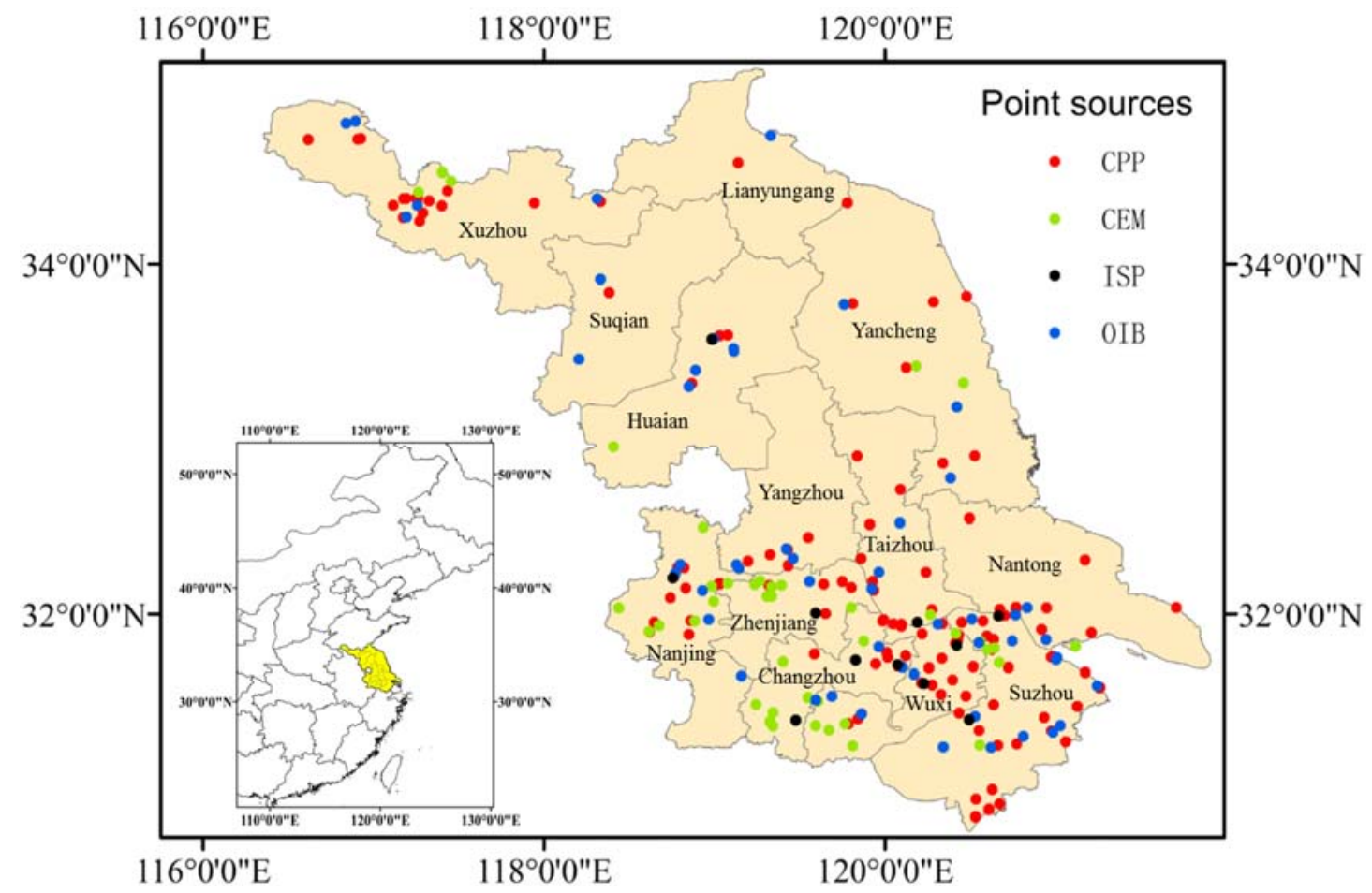


Figure S2

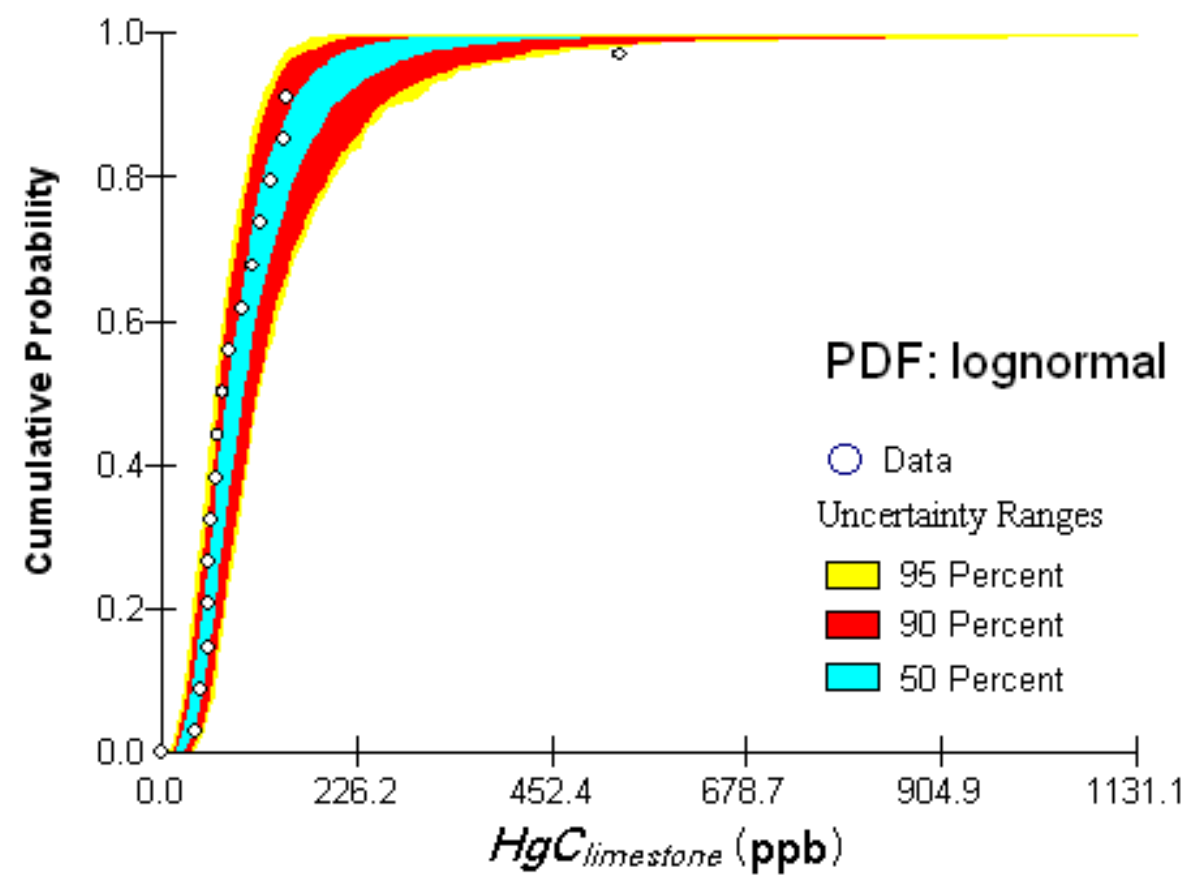


Figure S3

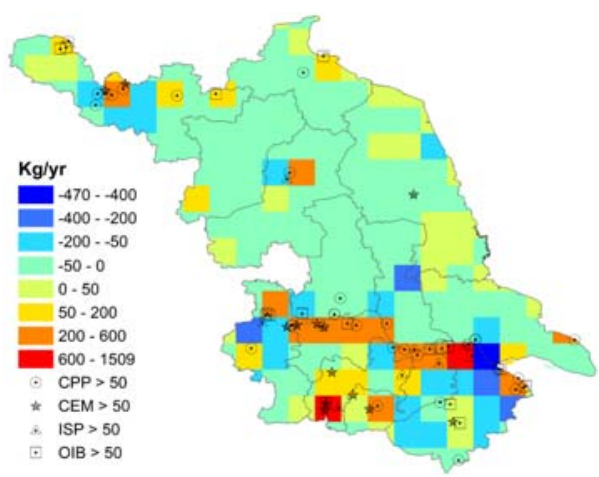

(a)

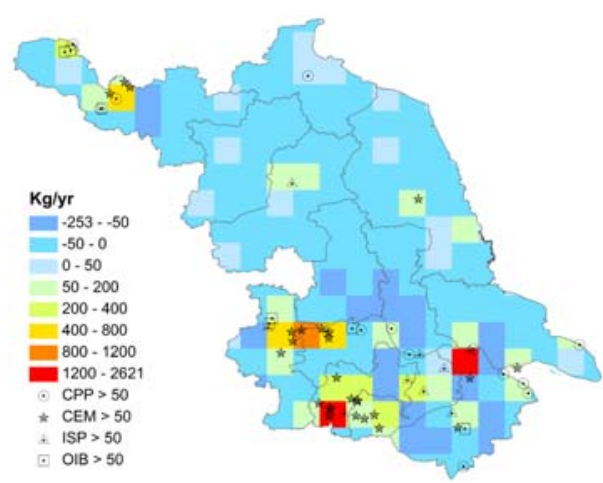

(d)

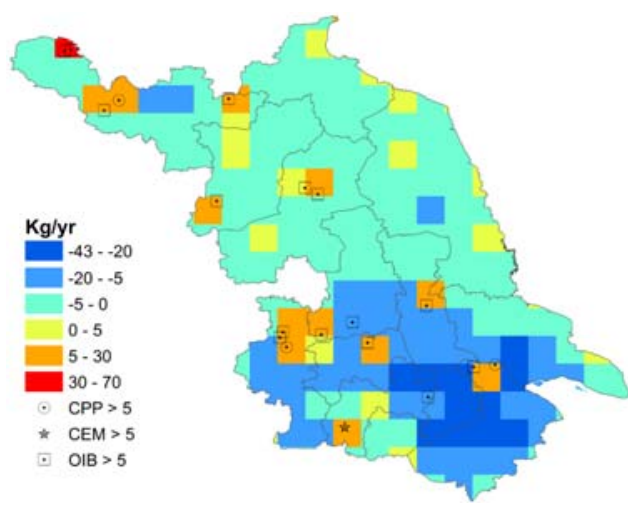

(h)

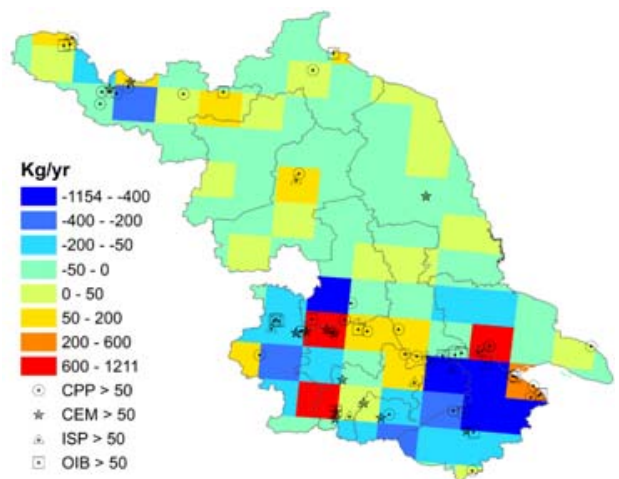

(b)

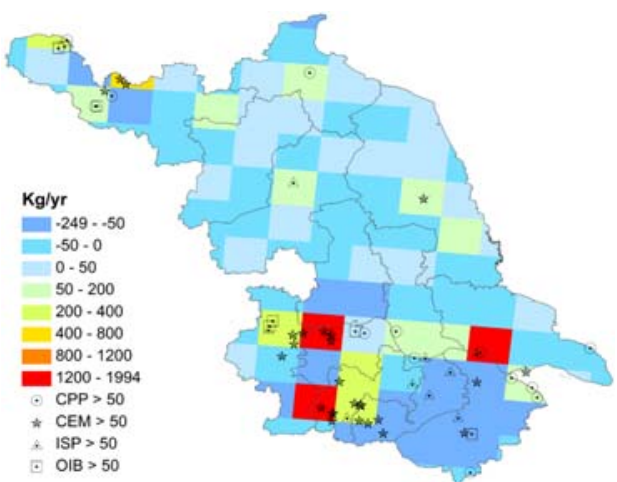

(e)

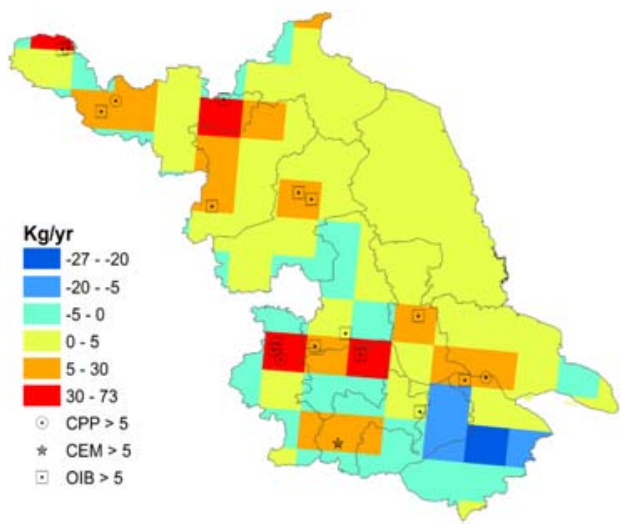

(i)

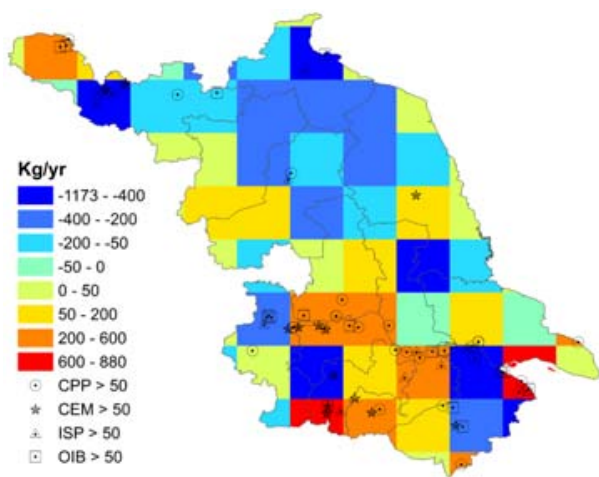

(c)

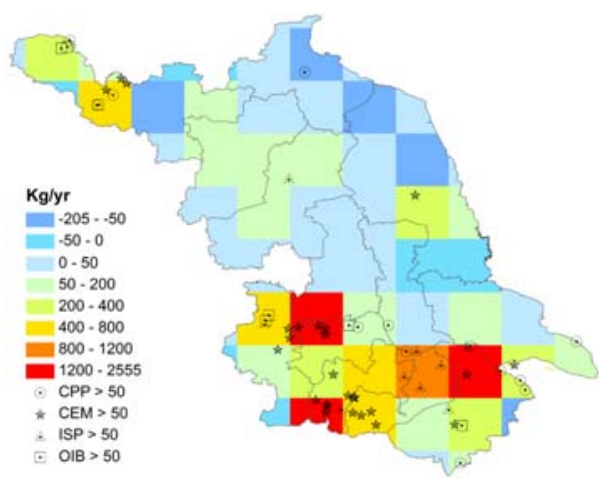

(f)

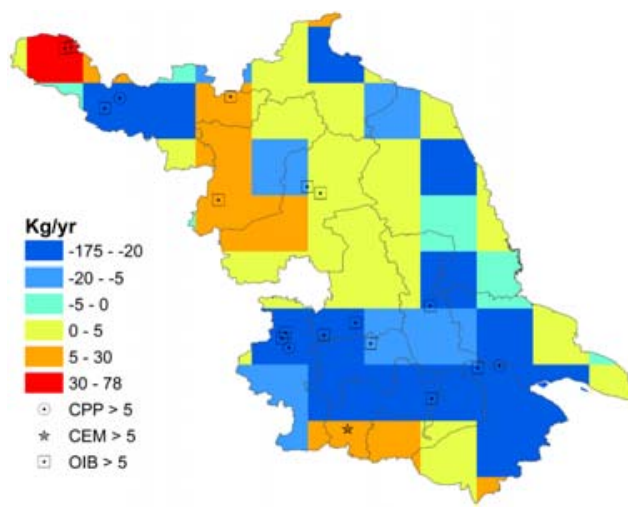

(j) 
Table S1 Summary of calculation methods of Hg emissions for coal-fired power plants (CPP), cement production (CEM), iron \& steel plants (ISP) and other industrial boilers (OIB) in multi-scale inventories.

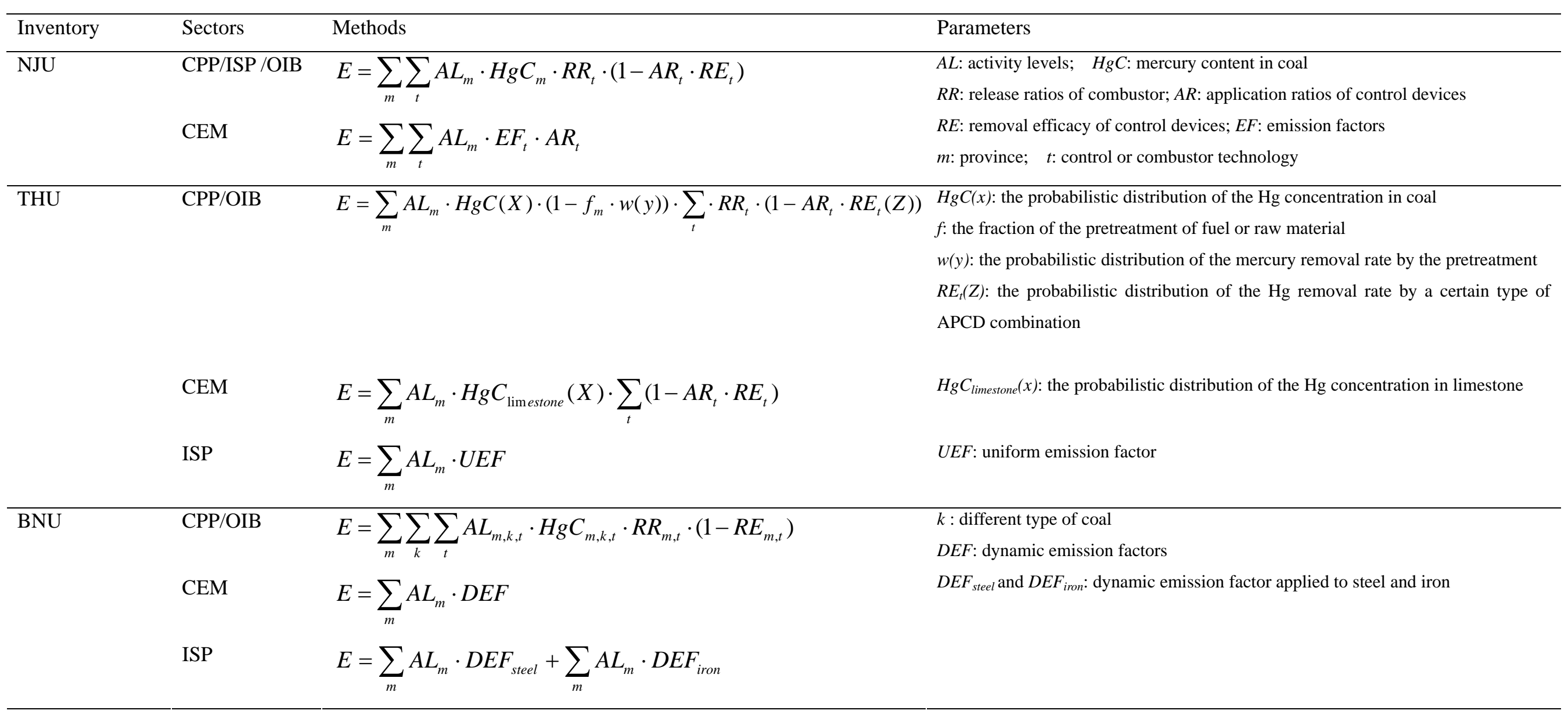




\section{Table S1 (continued)}

\begin{tabular}{|c|c|c|c|}
\hline Inventory & Sectors & Methods & Parameters \\
\hline AMAP/UNEP & $\begin{array}{l}\text { CPP/ISP/ } \\
\text { OIB/CEM }\end{array}$ & $E=\sum_{t} A L_{t} \cdot I E F \cdot\left(1-A R_{t} \cdot R E_{t}\right)$ & IEF: input emission factor \\
\hline EDGARv4.tox2 & CPP & $\begin{array}{l}E=\sum_{t} A L_{t} \cdot I E F \cdot\left(1-A R_{t} \cdot R E_{t}\right) \\
E=\sum A L \cdot U E F\end{array}$ & $\begin{array}{l}E F_{\text {sinter }}, E F_{\text {steel }} \text { and } E F_{\text {iron }} \text { : emission factor applied to sinter, steel and iron } \\
E F I_{I S P}, E F_{N M S} \text { and } E F_{\text {other: }} \text { emission factor for coal combustion in iron and pig } \\
\text { production, nonferrous metal smelting and other industry plants }\end{array}$ \\
\hline & ISP & $\begin{array}{l}E=\sum A L \cdot E F_{\text {sinter }}+\sum A L \cdot E F_{\text {iron }}+\sum A L \cdot E F_{\text {steel }} \\
E=\sum A L \cdot E F_{I S P}+\sum A L \cdot E F_{N M S}+\sum A L \cdot E F_{\text {other }}\end{array}$ & \\
\hline
\end{tabular}


Table S2 Database of $\mathrm{Hg}$ removal efficiencies by air pollutant control device (APCD).

\begin{tabular}{|c|c|c|}
\hline Control Device & Removal Efficiencies (\%) & References \\
\hline \multirow{21}{*}{$\mathrm{ESP}^{\mathrm{a}}$} & $10.78 ; 1.62$ & Wang et al. (2008b) \\
\hline & 13.5 & Zhou et al. (2008) \\
\hline & 24.4; 36.16; 43.33; 15.41 & Luo (2009) \\
\hline & 38.7 & Wang et al. (2008c) \\
\hline & $0.4 ; 32.78 ; 23.46$ & L Yang et al. (2007) \\
\hline & $10.8 ; 4.7 ; 33.1$ & Xu et al. (2010) \\
\hline & $31.1 ; 31.2 ; 24.5$ & Zhu et al. (2001) \\
\hline & $6.0 ; 20.0 ; 4.0$ & Wang et al. (2008a) \\
\hline & 24.8 & Duan et al. (2005) \\
\hline & 35.7 & X Yang et al. (2007) \\
\hline & $2.17 ; 8.75 ; 23.4$ & Wang et al. (2011) \\
\hline & $36.5 ; 46.4 ; 10.3 ; 6.1 ; 23.8$ & Wang et al. (2010a) \\
\hline & 25.2 & Li (2011) \\
\hline & $2.61 ; 5.83 ; 7.91 ; 15.1$ & C Wu et al. (2010) \\
\hline & 23.1; 23.8; 29.4; 17.8; 30.3; 32.2 & Chen et al. (2006) \\
\hline & $12.8 ; 17.4$ & Li et al. (2013) \\
\hline & 29.4 & Y Shi et al. (2013) \\
\hline & 46.63; 43.7; 55.9; 4.4; 37.92; 14.86; 13.49 & Wang et al. (2012) \\
\hline & 27.9 & X Shi et al. (2013) \\
\hline & 90 & $\mathrm{Hu}$ and $\mathrm{Fu}(2013)$ \\
\hline & 37 & Liu et al. (2014) \\
\hline \multirow{4}{*}{$\mathrm{ESP}^{\mathbf{b}}$} & 50.6 & L Yang et al. (2007) \\
\hline & 59.6 & Gao et al. (2007) \\
\hline & 18.5 & Wang et al. (2010a) \\
\hline & 55.9 & Wang (2013) \\
\hline \multirow{13}{*}{$\mathrm{ESP}+\mathrm{FGD}$} & 67.8; 70.0; 81.36; 27.5; 35.5 & Wang et al. (2010a) \\
\hline & $20.9 ; 33.9 ; 69.4 ; 70.6 ; 62.1 ; 74.2$ & $\mathrm{Hu}(2009)$ \\
\hline & $19.1 ; 2.1$ & Wang et al. (2008c) \\
\hline & 9.6 & L Yang et al. (2007) \\
\hline & 73.5; 84.9; 54.4 & Xu et al. (2010) \\
\hline & $62.3 ; 4.4 ; 95.9 ; 44.6$ & Wang et al. (2011) \\
\hline & $67.3 ; 78.3$ & Li (2011) \\
\hline & $62.2 ; 55.3 ; 69.5 ; 65.2$ & C Wu et al. (2010) \\
\hline & 68.7 & Shi et al. (2013) \\
\hline & $25.4 ; 38.4$ & Li et al. (2013) \\
\hline & 77.7; 93.2; 90.8; 86.9; 83.8; 97.4 & Xie and Yi (2014) \\
\hline & $53.2 ; 56.6$ & Wang (2013) \\
\hline & $65 ; 54$ & JSEMC (2013) \\
\hline
\end{tabular}


Table S2 (continued)

\begin{tabular}{|c|c|c|}
\hline Control Device & Removal Efficiencies (\%) & References \\
\hline \multirow{5}{*}{$\mathrm{FF}$} & 75.8 & L Yang et al. (2007) \\
\hline & $84.0 ; 20.7$ & Zhang et al. (2008) \\
\hline & $76.9 ; 23.0$ & Wang et al. (2008a) \\
\hline & 22.18 & He et al. (2012) \\
\hline & 29 & JSEMC (2013) \\
\hline \multirow{4}{*}{ WET } & 8.7 & Kilgroe et al. (2001) \\
\hline & 4.3 & JSEMC (2013) \\
\hline & 26 & Afonso and Senior (2001) \\
\hline & 8; 6.7; 10.6; 58.5 & Zhang (2012) \\
\hline \multirow{3}{*}{ CYC } & 0.1 & Kilgroe et al. (2002) \\
\hline & 12 & Huang et al. (2004) \\
\hline & 0 & \\
\hline \multirow{11}{*}{$\begin{array}{c}\text { SCR+FGD / } \\
\text { SCR+ESP+FGD }\end{array}$} & 82.2 & Li (2011) \\
\hline & 36.5 & Wang et al. (2010a) \\
\hline & 85 & Wang et al. (2010b) \\
\hline & 82.2 & Zhi et al. (2013) \\
\hline & 95 & Cheng et al. (2009) \\
\hline & 74.1 & Chen et al. (2008) \\
\hline & 81 & JSEMC (2013) \\
\hline & 70.86 & Zhang (2012) \\
\hline & 86; 80.84 & Wang (2013) \\
\hline & 85.4 & Xie and Yi (2014) \\
\hline & 73.55 & Fu (2013) \\
\hline \multirow{4}{*}{$\begin{array}{c}\text { FGD/ } \\
\text { FGD+WET }\end{array}$} & $24.3 ; 38$ & Zhong et al. (2010) \\
\hline & 75 & Hu and Fu (2013) \\
\hline & 59.4 & Gao et al. (2014) \\
\hline & 6 & Q Wang et al. (2008) \\
\hline $\mathrm{FF}+\mathrm{FGD}$ & 86 & Zhang (2012) \\
\hline $\mathrm{SCR}+\mathrm{FF}+\mathrm{FGD}$ & 93 & Zhang (2012) \\
\hline
\end{tabular}


Table S3 Database of mass fractions of speciated Hg by APCD.

\begin{tabular}{|c|c|c|}
\hline APCDs & $\begin{array}{l}\text { Mercury speciation (\%) } \\
\left(\mathrm{Hg}^{2+}, \mathrm{Hg}^{\mathrm{p}}, \mathrm{Hg}^{0}\right)\end{array}$ & References \\
\hline \multirow{12}{*}{ ESP } & $(11.4,0.0,88.6) ;(17.1,0.0,82.9)$ & Wang et al. (2008b) \\
\hline & $(53.5,0.1,46.4)$ & Zhou et al. (2008) \\
\hline & $(39.5,0.1,60.4)$ & Wang et al. (2008c) \\
\hline & $(11.4,0.0,88.6) ;(45.8,0.0,54.2) ;(8.1,0.0,91.9)$ & L Yang et al. (2007) \\
\hline & $(30.5,0.9,68.6) ;(30.2,0.0,69.8) ;(66.1,2.4,31.5)$ & Xu et al. (2010) \\
\hline & $(8.1,0.0,91.9) ;(44.7,0.0,55.3) ;(17.1,0.0,82.9)$ & Wang et al. (2008a) \\
\hline & $(74.0,0.0,26.0)$ & Duan et al. (2005) \\
\hline & $(7.5,0.0,92.5)$ & X Yang et al. (2007) \\
\hline & $\begin{array}{l}(67.5,0.1,32.4) ;(49.4,0.0,50.6) ;(64.0,0.0,36.0) \\
(14.3,0.7,85.0) ;(30.6,0.0,69.4)\end{array}$ & Wang et al. (2010a) \\
\hline & $\begin{array}{l}(34.0,0.9,65.1) ;(59.0,0.2,40.8) ;(79.8,0.0,20.2) ; \\
(75.8,0.0,24.2) ;(60.0,6.5,33.5) ;(30.8,10.4,33.5) ; \\
(45.8,3.0,51.2)\end{array}$ & Wang et al. (2012) \\
\hline & $(73.5,0,26.5) ;(83.6,0.1,16.3) ;(27.5,0.6,71.9)$ & Zhang (2012) \\
\hline & $(16.2,15.7,68.1)$ & X Shi et al. (2013) \\
\hline \multirow{4}{*}{$\mathrm{ESP}+\mathrm{FGD}$} & $\begin{array}{l}(24.8,0.0,75.2) ;(18.3,0.0,81.7) ;(8.9,0.0,91.1) ; \\
(6.2,0.0,93.8) ;(11.5,0.0,88.5)\end{array}$ & Wang et al. (2010a) \\
\hline & $(5.4,0.0,94.6) ;(3.9,0,96.1)$ & Wang et al. (2008b) \\
\hline & $(4.0,0.0,96.0)$ & L Yang et al. (2007) \\
\hline & $(27.7,0.6,71.7) ;(14.9,0.0,85.1) ;(53.7,2.5,43.8)$ & Xu et al. (2010) \\
\hline \multirow{5}{*}{ FF } & $(65.4,24.5,10.1)$ & Zhang et al. (2008) \\
\hline & $(74.5,8.8,16.7) ;(75.6,0.0,24.4)$ & Wang et al. (2008a) \\
\hline & $(78,10,12)$ & L Yang et al. (2007) \\
\hline & $(33,0,64) ;(36,1,63)$ & Zhang (2012) \\
\hline & $(74,2,24)$ & He et al. (2012) \\
\hline \multirow{4}{*}{$\begin{array}{l}\text { SCR+ESP / } \\
\text { ESP+FGD }\end{array}$} & $(10.7,0.0,89.5)$ & Wang et al. (2010a) \\
\hline & $\begin{array}{l}(20.9,0.0,79.1) ;(15.6,0.0,84.4) \\
(16.6,0.0,83.4) ;(17.7,0.0,82.3)\end{array}$ & Zhong et al. (2010) \\
\hline & $(84.1,0.0,15.9)$ & Chen et al. (2008) \\
\hline & $(34.6,0.4,65.0)$ & Zhang (2012) \\
\hline WET & $\begin{array}{l}(9.7,2.9,87.4) ;(16.2,3.8,80.0) ;(59.1,0.2,39.9) ; \\
(60.4,0.2,39.4) ;(18.4,0.5,81.1) ;(30.7,4.7,64.6)\end{array}$ & Zhang (2012) \\
\hline $\mathrm{FF}+\mathrm{FGD}$ & $(78,21,1)$ & Zhang (2012) \\
\hline
\end{tabular}


Table S4 Emission factors and Hg speciation profiles for ISP and other selected sources in Categories 2 and 3.

\begin{tabular}{|c|c|c|c|c|c|c|c|}
\hline \multirow{2}{*}{\multicolumn{2}{|c|}{ Sources }} & \multirow{2}{*}{$\mathrm{EF}$} & \multirow{2}{*}{ Unit } & \multirow{2}{*}{ References } & \multicolumn{3}{|c|}{ Speciation/ \% } \\
\hline & & & & & $\mathrm{Hg}^{0}$ & $\mathrm{Hg}^{2+}$ & $\mathrm{Hg}^{\mathrm{p}}$ \\
\hline \multirow{2}{*}{\multicolumn{2}{|c|}{ ISP }} & $0.042^{\mathrm{a}}$ & g/t crude & \multirow{2}{*}{ Wang et al. (2016) } & \multirow{2}{*}{34} & \multirow{2}{*}{66} & \multirow{2}{*}{0} \\
\hline & & $0.068^{\mathrm{b}}$ & steel & & & & \\
\hline \multicolumn{2}{|r|}{ MSWI } & 0.22 & $g / t$ & $\begin{array}{l}\text { Chen et al. (2013); } \\
\text { Hu et al. (2012) }\end{array}$ & 13 & 86 & 1 \\
\hline \multicolumn{2}{|r|}{ BFLP } & 0.035 & t/t Hg used & AMAP/UNEP (2013) & 100 & 0 & 0 \\
\hline \multicolumn{2}{|r|}{ PVC } & 0.01 & t/t Hg used & THU (2009) & 100 & 0 & 0 \\
\hline \multicolumn{2}{|r|}{$\mathrm{HC}$} & 1 & g/corpse & UNEP (2005) & 96 & 0 & 4 \\
\hline \multicolumn{2}{|c|}{ NMS (Cu) } & 0.4 & $\mathrm{~g} / \mathrm{t} \mathrm{Cu}$ & Zhao et al. (2015) & 50 & 50 & 0 \\
\hline \multicolumn{2}{|r|}{$\mathrm{AP}$} & 0.265 & $g / t$ & THU (2006) & 80 & 15 & 5 \\
\hline \multicolumn{2}{|r|}{ Heavy fuel } & 0.014 & $g / t$ & Wu et al. (2006) & & & \\
\hline \multirow[t]{2}{*}{$O \& G$} & Oil & 0.058 & $g / t$ & Wu et al. (2006) & 50 & 40 & 10 \\
\hline & Gas & 0.416 & $g / t$ & Wu et al. (2006) & & & \\
\hline \multirow{2}{*}{$\mathrm{BIO}$} & Crop & 16.7 & $\mathrm{ng} / \mathrm{g}$ & \multirow{2}{*}{$\begin{array}{l}\text { Huang et al. (2011); } \\
\text { Zhang et al. (2013) }\end{array}$} & \multirow{2}{*}{76} & \multirow{2}{*}{19} & \multirow{2}{*}{5} \\
\hline & Fuel wood & 12.3 & $\mathrm{ng} / \mathrm{g}$ & & & & \\
\hline
\end{tabular}

${ }^{\mathrm{a}}$ Including mercury emissions from rotary kiln for limestone and dolomite; ${ }^{\mathrm{b}}$ Excluding mercury emissions from rotary kiln for limestone and dolomite 
Table S5 Removal efficiencies and application ratios of various APCDs between multi-scale Hg emission inventories.

\begin{tabular}{|c|c|c|c|c|c|}
\hline & APCDs & Bottom-up & NJU & THU & AMAP/UNEP \\
\hline \multirow{10}{*}{$\begin{array}{l}\text { Removal } \\
\text { efficiency }\end{array}$} & CYC & 4.0 & 4.0 & & \\
\hline & WET & 17.5 & 13.0 & 23.0 & \\
\hline & ESP & 23.5 & 20.3 & 29.0 & 26.0 \\
\hline & FGD+WET & 39.7 & & & \\
\hline & $\mathrm{CFB}+\mathrm{ESP}$ & 46.1 & 42.9 & & \\
\hline & $\mathrm{FF}$ & 48.6 & 56.1 & 67.0 & 50.0 \\
\hline & ESP+FGD & 58.8 & 53.8 & 62.0 & 65.0 \\
\hline & $\mathrm{SCR}+\mathrm{ESP}+\mathrm{FGD}$ & 77.7 & 76.6 & 69.0 & 69.0 \\
\hline & $\mathrm{FF}+\mathrm{FGD}$ & 86.4 & & 86.0 & \\
\hline & $\mathrm{SCR}+\mathrm{FF}+\mathrm{FGD}$ & 93.0 & & 93.0 & \\
\hline \multirow{10}{*}{$\begin{array}{l}\text { Application } \\
\text { ratio for CPP }\end{array}$} & CYC & 0.0 & 1.5 & 0.0 & 0.0 \\
\hline & WET & 4.5 & 1.3 & 0.0 & 0.0 \\
\hline & ESP & 3.5 & 13.6 & 10.2 & 7.0 \\
\hline & FGD+WET & 2.5 & 0.0 & 0.0 & 0.0 \\
\hline & CFB+ESP & 4.0 & 4.0 & 0.0 & 0.0 \\
\hline & $\mathrm{FF}$ & 2.5 & 6.8 & 0.8 & 7.0 \\
\hline & ESP+FGD & 70.0 & 61.6 & 69.8 & 72.0 \\
\hline & SCR+ESP+FGD & 11.4 & 11.2 & 11.2 & 14.0 \\
\hline & $\mathrm{FF}+\mathrm{FGD}$ & 0.2 & 0.0 & 5.2 & 0.0 \\
\hline & $\mathrm{SCR}+\mathrm{FF}+\mathrm{FGD}$ & 1.3 & 0.0 & 0.8 & 0.0 \\
\hline \multirow{10}{*}{$\begin{array}{l}\text { Application } \\
\text { ratio for OIB }\end{array}$} & CYC & 9.9 & 29.5 & 0.0 & \\
\hline & WET & 26.9 & 41.9 & 95.0 & 50.0 \\
\hline & ESP & 13.3 & 5.1 & 0.0 & \\
\hline & FGD+WET & 4.0 & 0.0 & 0.0 & 0.0 \\
\hline & $\mathrm{CFB}+\mathrm{ESP}$ & 6.0 & 0.0 & 0.0 & 0.0 \\
\hline & $\mathrm{FF}$ & 10.0 & 23.4 & 0.0 & 25.0 \\
\hline & ESP+FGD & 10.8 & 0.0 & 0.0 & 0.0 \\
\hline & SCR+ESP+FGD & 2.6 & 0.0 & 0.0 & 0.0 \\
\hline & $\mathrm{FF}+\mathrm{FGD}$ & 1.5 & 0.0 & 5.0 & 0.0 \\
\hline & No & 15.0 & 0.0 & 0.0 & 25.0 \\
\hline
\end{tabular}


Table S6 Uncertainties of Hg emission factors for main sources, expressed as the probability distribution functions (PDF).

\begin{tabular}{|c|c|c|c|c|c|}
\hline \multirow{2}{*}{ Parameters } & \multirow{2}{*}{ Samples } & \multirow{2}{*}{ Distribution } & \multicolumn{3}{|c|}{ Key characteristics for distribution functions } \\
\hline & & & $\mathrm{P} 10^{\mathrm{a}} / \mathrm{Min}^{\mathrm{b}}$ & $\mathrm{P} 90^{\mathrm{a}} / \mathrm{Max}^{\mathrm{b}}$ & P50 /Most likely ${ }^{\mathrm{b}}$ \\
\hline \multicolumn{6}{|c|}{ Mercury content in raw coal produced in given province for CPP/OIB/RCC , g/t } \\
\hline Anhui & 20 & Lognormal & 0.08 & 0.38 & 0.15 \\
\hline Gansu & 7 & Lognormal & 0.04 & 0.17 & 0.07 \\
\hline Hebei & 24 & Lognormal & 0.04 & 0.32 & 0.11 \\
\hline Heilongjiang & 20 & Lognormal & 0.02 & 0.09 & 0.04 \\
\hline Henan & 37 & Lognormal & 0.06 & 0.35 & 0.17 \\
\hline Inner Mongolia & 46 & Lognormal & 0.02 & 0.4 & 0.09 \\
\hline Jiangsu & 11 & Lognormal & 0.11 & 0.6 & 0.2 \\
\hline Shaanxi & 28 & Lognormal & 0.02 & 0.53 & 0.08 \\
\hline Shandong & 33 & Lognormal & 0.05 & 0.28 & 0.12 \\
\hline Shanxi & 88 & Lognormal & 0.03 & 0.36 & 0.09 \\
\hline \multicolumn{6}{|c|}{ Release rates of boilers for CPP, \% } \\
\hline PC & 32 & Triangular & 89 & 100 & 99 \\
\hline Grate & 2 & Triangular & 92 & 100 & 96 \\
\hline CFB & 3 & Triangular & 93 & 100 & 98 \\
\hline \multicolumn{6}{|c|}{ Release rates of boilers for OIB/HB/FOS, \% } \\
\hline Grate & 3 & Triangular & 51 & 91 & 76 \\
\hline CFB & 1 & Triangular & 51 & 100 & 91 \\
\hline \multicolumn{6}{|c|}{ Hg removal efficiency by APCDs for CPP and CEM, \% } \\
\hline $\mathrm{FF}$ & 7 & Weibull & 21 & 84 & 49 \\
\hline ESP & 54 & Normal & 21 & 27 & 24 \\
\hline WET & 6 & Weibull & 11 & 26 & 18 \\
\hline FGD+ESP & 40 & Weibull & 44 & 70 & 59 \\
\hline CYC & 3 & Uniform & 0 & 14 & - \\
\hline SCR & 12 & Weibull & 70 & 84 & 78 \\
\hline $\mathrm{CFB}+\mathrm{ESP}$ & 4 & Weibull & 33 & 60 & 46 \\
\hline $\mathrm{DPT}+\mathrm{DR}$ & 3 & Uniform & 2 & 10 & - \\
\hline SKT/RKT+ESP & 2 & Triangular & 10 & 80 & 22 \\
\hline $\mathrm{SKT} / \mathrm{RKT}+\mathrm{FF}$ & 2 & Triangular & 21 & 84 & 80 \\
\hline \multicolumn{6}{|c|}{ Hg content in raw materials for CEM, ppb } \\
\hline limestone & & Lognormal & 81 & 123 & 101 \\
\hline $\begin{array}{l}\text { other raw materials } \\
\text { Iron \& Steel plants }\end{array}$ & 1 & Normal & 51 & 65 & 58 \\
\hline $\mathrm{EF}_{\text {with raw material production }}$ & 1 & Uniform & 0.04 & 0.1 & - \\
\hline $\mathrm{EF}_{\text {without raw material production }}$ & 1 & Uniform & 0.03 & 0.07 & - \\
\hline \multicolumn{6}{|c|}{ Biofuel use/biomass open burning } \\
\hline Firewood & 26 & Uniform & 0 & 50 & - \\
\hline Crops & 9 & Uniform & 0 & 106 & - \\
\hline \multicolumn{6}{|l|}{ Waste incineration } \\
\hline Municipal & 29 & Weibull & 0.21 & 0.32 & 0.27 \\
\hline Release rates & 1 & Normal & 0.37 & 0.63 & 0.5 \\
\hline Hg content & 31 & Weibull & 0.12 & 1.58 & 0.6 \\
\hline
\end{tabular}


${ }^{\text {a }} \mathrm{P} 10$ values mean that there is a probability of $10 \%$ that the actual result would be equal to or below the P10 values; P50 mean that there is a probability of 50\% that the actual result would be equal to or below the P50 values; and P90 mean that there is a probability of $90 \%$ that the actual result would be equal to or below the P90 values.

${ }^{\mathrm{b}}$ These values are for the minimum, the most likely, and the maximum values for the triangular distribution function instead of P10, P50, and P90 values, or for the minimum and maximum values for the uniform distribution function instead of P10 and P90 values. 
Table S7 Uncertainties of mass fractions of $\mathbf{H g}$ speciation for main source categories, expressed as the probability distribution functions (PDF).

\begin{tabular}{|c|c|c|c|c|c|c|}
\hline \multicolumn{2}{|c|}{ Parameters } & \multirow{3}{*}{$\begin{array}{c}\text { Samples } \\
7\end{array}$} & \multirow{3}{*}{$\begin{array}{c}\text { Distribution } \\
\text { Triangular }\end{array}$} & \multicolumn{3}{|c|}{ Key characteristics for distribution functions / \% } \\
\hline & & & & \multirow{2}{*}{$\frac{\text { P10/Min }}{10}$} & \multirow{2}{*}{$\frac{\text { P90a/Max }}{64}$} & \multirow{2}{*}{$\begin{array}{c}\text { P50/Most likely } \\
31\end{array}$} \\
\hline & $\mathrm{Hg}^{0}$ & & & & & \\
\hline$F F$ & $\mathrm{Hg}^{\mathrm{p}}$ & 7 & Triangular & 0 & 25 & 7 \\
\hline \multirow{2}{*}{ ESP } & $\mathrm{Hg}^{2+}$ & 31 & Weibull & 30 & 52 & 41 \\
\hline & $\mathrm{Hg}^{\mathrm{p}}$ & 31 & Triangular & 0 & 15 & 1 \\
\hline \multirow{2}{*}{ FGD+ESP } & $\mathrm{Hg}^{2+}$ & 11 & Triangular & 4 & 54 & 16 \\
\hline & $\mathrm{Hg}^{\mathrm{p}}$ & 11 & Triangular & 0 & 1 & 0.3 \\
\hline \multirow{2}{*}{ WET } & $\mathrm{Hg}^{0}$ & 6 & Uniform & 10 & 60 & 33 \\
\hline & $\mathrm{Hg}^{\mathrm{p}}$ & 6 & Uniform & 0.2 & 5 & 2 \\
\hline \multirow{2}{*}{$\mathrm{NOC}^{\mathrm{a}}$} & $\mathrm{Hg}^{0}$ & 37 & Triangular & 0 & 89 & 48 \\
\hline & $\mathrm{Hg}^{\mathrm{p}}$ & 37 & Gamma & 0.02 & 50 & 6 \\
\hline \multirow{2}{*}{ SCR } & $\mathrm{Hg}^{2+}$ & 7 & Triangular & 11 & 84 & 29 \\
\hline & $\mathrm{Hg}^{\mathrm{p}}$ & 7 & Triangular & 0 & 0.5 & 0.1 \\
\hline \multirow{2}{*}{$\mathrm{DPT}+\mathrm{DR}$} & $\mathrm{Hg}^{0}$ & 3 & Triangular & 9 & 39 & 23.8 \\
\hline & $\mathrm{Hg}^{\mathrm{p}}$ & 3 & Triangular & 0 & 1.6 & 0.5 \\
\hline ISP & $\mathrm{Hg}^{0}$ & 2 & Triangular & 25 & 78 & 34 \\
\hline \multirow{2}{*}{ BIO } & $\mathrm{Hg}^{0}$ & 25 & Weibull & 57.3 & 94.2 & 76.9 \\
\hline & $\mathrm{Hg}^{2+}$ & 25 & Triangular & 0 & 21.7 & 5 \\
\hline \multirow{2}{*}{ SWI } & $\mathrm{Hg}^{0}$ & 10 & Gamma & 1.1 & 33.8 & 6.2 \\
\hline & $\mathrm{Hg}^{\mathrm{p}}$ & 10 & Gamma & 0.1 & 2.6 & 0.5 \\
\hline
\end{tabular}

${ }^{\mathrm{a}}$ No control device for coal combustion 
Table S8 The parameters contributing most to emission uncertainties by sector for 2010. The percentages indicate the contributions of the parameters to the variance of corresponding emission estimates.

\begin{tabular}{|c|c|c|c|c|}
\hline & $\mathrm{HgT}$ & $\mathrm{Hg}^{0}$ & $\mathrm{Hg}^{2+}$ & $\mathrm{Hg}^{\mathrm{p}}$ \\
\hline \multirow[t]{3}{*}{ СРP } & $H g C_{\text {Shaanxi }} 26 \%$ & $H g C_{\text {Shaanxi }} 23 \%$ & $F_{E S P+F G D \_H g}{ }^{2+} 33 \%$ & $F_{E S P_{-} H g}^{p} 35 \%$ \\
\hline & $R E_{E S P+F G D} 20 \%$ & $R E_{E S P+F G D} 21 \%$ & $H g C_{\text {Shaanxi }} 19 \%$ & $H g C_{\text {Shaanxi }} 15 \%$ \\
\hline & $H g C_{\text {Inner Mongolia }} 18 \%$ & $H g C_{\text {Inner Mongolia }} 16 \%$ & $H g C_{\text {Inner Mongolia }} 12 \%$ & $H g C_{\text {Inner Mongolia }} 10 \%$ \\
\hline \multirow[t]{3}{*}{ CEM } & $H g C_{\text {limestone }} 84 \%$ & $F_{D P T+D R \_H g}{ }^{0} 66 \%$ & $H g C_{\text {limestone }} 65 \%$ & $F_{D P T+D R \_H g}^{p} 90 \%$ \\
\hline & $A l_{i} 7 \%$ & $H g C_{\text {limestone }} 27 \%$ & $F_{D P T+D R \_H g}{ }^{0} 21 \%$ & $H g C_{\text {limestone }} 6 \%$ \\
\hline & $R E_{D P T+D R} 3 \%$ & $A l_{i} 3 \%$ & $A l_{i} 5 \%$ & / \\
\hline \multirow[t]{3}{*}{ ISP } & $E F_{\text {limestone and dolomite }} 60 \%$ & $F_{H g}^{0} 66 \%$ & $F_{H g}{ }^{0} 59 \%$ & \\
\hline & $A l_{i} 24 \%$ & $E F_{\text {limestone and dolomite }} 24 \%$ & $E F_{\text {limestone and dolomite }} 28 \%$ & / \\
\hline & / & $A l_{i} 7 \%$ & $A l_{i} 9 \%$ & \\
\hline \multirow[t]{3}{*}{ OIB } & $H g C_{\text {Shaanxi }} 15 \%$ & $H g C_{\text {Shaanxi }} 10 \%$ & $H g C_{\text {Shaanxi }} 12 \%$ & $F_{\text {NOC_Hg }}^{p} 30 \%$ \\
\hline & $H g C_{\text {Inner Mongolia }} 11 \%$ & $H g C_{\text {Inner Mongolia }} 13 \%$ & $H g C_{\text {Inner Mongolia }} 8 \%$ & $H g C_{\text {Shaanxi }} 5 \%$ \\
\hline & $R_{\text {Grate }} 5 \%$ & $R R_{\text {Grate }} 3 \%$ & $R R_{\text {Grate }} 3 \%$ & $R R_{\text {Grate }} 2 \%$ \\
\hline \multirow[t]{3}{*}{ Rest } & $E F_{M S W I} 37 \%$ & $E F_{\text {crop }} 46 \%$ & $E F_{M S W I} 70 \%$ & $E F_{\text {crop }} 55 \%$ \\
\hline & $E F_{\text {crop }} 31 \%$ & $E F_{\text {oil }} 11 \%$ & $E F_{\text {oil }} 9 \%$ & $F_{\text {crop_Hg }}^{p} 20 \%$ \\
\hline & $E F_{\text {oil }} 16 \%$ & $R R_{I U S} 7 \%$ & $F_{\text {oil_Hg2+ }} 6 \%$ & $E F_{o i l} 8 \%$ \\
\hline \multirow[t]{4}{*}{ Total } & $H g C_{\text {Shaanxi }} 12 \%$ & $H g C_{\text {Shaanxi }} 12 \%$ & $H g C_{\text {Shaanxi }} 8 \%$ & $F_{\mathrm{NOC} \_H g}^{p} 18 \%$ \\
\hline & $H g C_{\text {Inner Mongolia }} 8 \%$ & $H g C_{\text {Inner Mongolia }} 8 \%$ & $\mathrm{HgC}_{\text {limestone }} 8 \%$ & $E F_{\text {crop }} 9 \%$ \\
\hline & $\mathrm{HgC}_{\text {jiangsu }} 7 \%$ & $\mathrm{HgC}_{\text {jiangsu }} 7 \%$ & $H g C_{\text {Inner Mongolia }} 5 \%$ & $F_{\text {crop_Hg }}^{p} 5 \%$ \\
\hline & $R E_{E S P+F G D} 4 \%$ & $R E_{E S P+F G D} 6 \%$ & $\mathrm{HgC}_{\text {jiangsu }} 4 \%$ & $\mathrm{HgC}_{\text {Shaanxi }} 4 \%$ \\
\hline
\end{tabular}




\section{References}

AMAP/UNEP: Technical Background Report to the Global Atmospheric Mercury Assessment, Arctic Monitoring and Assessment Programme, Oslo, Norway/UNEP Chemicals Branch, Geneva, Switzerland, 159 pp., 2008

AMAP/UNEP: Technical Background Report for the Global Mercury Assessment, Arctic Monitoring and Assessment Programme, Oslo, Norway/UNEP Chemicals Branch, Geneva, Switzerland, 263 pp., 2013.

Afonso, R. F., Senior, C. L.: Assessment of mercury emissions from full scale power plants, Proceedings of the EPRI-EPA-DOE-AWMA mega symposium and mercury conference, Chicago, IL, 2001.

Chen, J., Yuan, D., Li, Q., Zheng, J., Zhu, Y., Hua, X., He, S., Zhou, J.: Effect of flue-gas cleaning devices on mercury emission from coal-fired boiler, Proceedings of the CSEE, 02, 72-76, 2008 (in Chinese).

Chen, L., Liu, M., Fan, R., Ma, S., Xu, Z., Ren, M., He, Q.: Mercury speciation and emission from municipal solid waste incinerators in the Pearl River Delta, South China. Sci. Total Environ., 447, 396-402, 2013

Chen, Y., Chai, F., Xue, Z., Liu, T., Chen, Y., Tian, C.: Study on mercury emission factors for coal-fired power plants, Research of Environmental Sciences, 02, 49-52, 2006 (in Chinese).

Cheng, C., Hack, P., Chu, P., Chang, N., Lin, T.: Partitioning of mercury, arsenic, selenium, boron, and chloride in a full-scale coal combustion process equipped with selective catalytic reduction, electrostatic precipitation, and flue gas desulfurization systems, Energy Fuels, 10, 4805-4816, 2009.

Chu, P., Porcella, D. B.: Mercury stack emissions from US electric utility power plants, Water Air Soil Pollut., 80, 135-144, 1995.

Duan, Y., Cao, Y., Kellie, S., Liu, K., Riley, J., Pan, W.: In-situ measurement and distribution of flue gas mercury for a utility PC boiler system, Journal of Southeast University (English Edition), 01, 53-57, 2005.

Fu, J.: Study on characterization of atmospheric mercury emissions from a coal-fired power plant in Beijing and emission factor(Master Thesis), Chengdu University of technology, Chengdu, China, 2013 (in Chinese).

Gao, H., Wang, X., Zhou, J., Luo, Z.: The influence of ESP on mercury emission from coal-fired power plant, Boiler Technology, 05, 63-67, 2007 (in Chinese).

Gao, Z., Wu, P., Chen, S., Fan, Y., Yin, L.: Mercury removal performance of wet flue gas desulfurization system in field test, Chinese Journal of Environmental Engineering, 08, 3887-3892, 2014.

He, Z., Kan, Z., Qi, L., Han, X.: Analysis to mercury removal performance test of bag-type dust collector, Inner Mongolia Electric Power 30, 0040-0042, 2012 (in Chinese).

Hu, D., Zhang, W., Chen, L., Chen, C., Ou, L., Tong, Y., Wei, W., Long, W., Wang, X.: Mercury emissions from waste combustion in China from 2004 to 2010, Atmos. Environ., 62, 359-366, 2012.

Hu, J., Fu, C.: Mercury emission characteristics from coal-fired 300 MW generating units based on measurements, Journal of Natural Science of Hunan Normal University, 36, 83-87, 2013.

Hu, Y.: Mercury emission of coal combustion and analysis of mercury removal technology at home and abroad, Environment Science and Technology, 03, 69-72, 2011 (in Chinese).

Huang, X., Li, M., Friedli, H. R. Song, Y., Chang, D., Zhu, L.: Mercury emissions from biomass burning in China, Environ. Sci. Technol., 45, 9442-9448, 2011.

Huang, Y. J., Jin, B. S., Zhong, Z. P., Xiao. R.: Study on the distribution of trace elements in gasification products, Proceedings of the CSEE, 11, 208-2012, 2004 (in Chinese). 
Jiangsu Environment Monitoring Center (JSEMC): Research on mercury emissions in flue gas of coal-fired power plants in Jiangsu Province, Interim report, 2013 (in Chinese).

Kilgroe, J., Sedman, C., Srivastava, R., Ryan, J., Lee, C., Thorneloe, S.: Control of mercury emissions from coal-fired electric utility boilers: interim report including errata dated 3-21-02, EPA-600/R-01-109, 2002.

Li, G., Feng, X., Li, Z., Qiu, G., Shang, L., Liang, P., Wang, D., Yang, Y.: Mercury emission to atmosphere from primary Zn production in China, Sci. Total Environ., 408, 4607-4612, 2010.

Li, W.: Characterization of atmospheric mercury emissions from coal-fired power plant and cement plant(Master Thesis), Xi'nan University, Chongqing, China, 2011 (in Chinese).

Li, Z., Duan, Y.: Mercury removal by ESP and WFGD in a 300 MW coal-fired power plant, Journal of Fuel Chemistry and Technology, 04, 0491-0498, 2013 (in Chinese).

Liu, J., Xue, J., Xu, Y., Wang, H., Chen, S.: Control of mercury emission in coal fired plants by electrostatic precipitator synergy, Chinese Journal of Environmental Engineering, 08, 4853-4857, 2014 (in Chinese).

Luo, G. Q: Study of species identification and removal of mercury in coal and during coal combustion(Master Thesis), Huazhong University of Science and Technology, Wuhan, China, 2009 (in Chinese).

Muntean, M., Jassens-Maenhout, G., Song, S., Selin, N. E., Olivier, J. G. J., Guizzardi, D., Maas, R., and Dentener, F.: Trend analysis from 1970 to 2008 and model evaluation of EDGARv4 global gridded anthropogenic mercury emissions, Sci. Total Environ., 494-495, 337-350, 2014.

Pacyna, E. G., Pacyna, J. M., Sundseth, K., Munthe, J., Kindbom, K., Wilson, S., Steenhuisen, F., Maxson, P.: Global emission of mercury to the atmosphere from anthropogenic sources in 2005 and projections to 2020, Atmos. Environ., 44, 2487-2499, 2010.

Rafaj, P., Bertok, I., Cofala, J., and Schopp, W.: Schopp, W.: Scenarios of global mercury emissions from anthropogenic sources, Atmos. Environ., 79, 472-479, 2013.

Shi, X., Wu, J., Lu, P., Zhang, J., Ren, J., Shi, Z., Li, X.: Experimental Study on the Flue Gas Mercury Removal Capability of ESP, East China Electric Power, 41, 0459-0462, 2013.

Shi, Y., Deng, S., Zhang, F., Deng, Z., Cao, Q., Liu, Y., Shu, X.: Effect of flue gas pollution control devices on mercury emission from coal-fired power plants, Advanced Materials Research, 726, 2160-2164, 2013.

Streets, D. G., Hao, J., Wu, Y. Jiang, J., Chan, M., Tian, H., Feng, X.: Anthropogenic mercury emissions in China, Atmos. Environ., 39, 7789-7806, 2005.

Tian, H. Z., Wang, Y., Xue, Z. G., Cheng, K., Qu, Y. P., Chai, F. H., Hao, J. M.: Trend and characteristics of atmospheric emissions of $\mathrm{Hg}$, As, and Se from coal combustion in China, 1980-2007, Atmos. Chem. Phys., 10, 11905-11919, 2010.

Tian, H., Wang, Y., Cheng, K., Qu, Y., Hao, J, Xue, Z., Chai, F.: Control strategies of atmospheric mercury emissions from coal-fired power plants in China, J. Air Waste Manag. Assoc., 62, 576-58, 2012.

Tsinghua University (THU): Improve the Estimates of Anthropogenic Mercury Emissions in China, Technical report, 2009. Available online at: http://ww.chem.unep.ch/MERCURY/.

Tsinghua University (THU): Improve the Estimates of Anthropogenic Mercury Emissions in China; United Nations Environment Programme (UNEP): Geneva, Switzerland, 2006.

U.S. Environmental Protection Agency (USEPA):Mercury study report to congress. EPA-452/R-97-003, Washington DC: USEPA, 1997.

United Nations Environment Programme (UNEP). Toolkit for identification and quantification of mercury releases; UNEP Chemicals Branch, 2005. 
Wang, F. Y., Wang S. X., Zhang, L., Yang, H., Gao, W., Wu, Q. R., Hao, J. M.: Mercury mass flow in iron and steel production process and its implications for mercury emission control, Journal of Environmental Sciences, 2016, available at: http://dx.doi.org/10.1016/j.jes.2015.07.019.

Wang, J., Wang, W., Xu, W., Wang, X., Zhao, S.: Mercury removals by existing pollutants control devices of four coal-fired power plants in China, Journal of Environmental Sciences, 11, 1839-1844, 2011.

Wang, S. X., Zhang, L., Li, G. H., Wu, Y., Hao, J. M., Pirrone, N., Sprovieri, F., Ancora, M. P.: Mercury emission and speciation of coal-fired power plants in China, Atmos. Chem. Phys., 10, 1183-1192, 2010a.

Wang, S. X., Zhang, L., Wu, Y., Ancora, M. P., Zhao, Y., Hao, J. M.: Synergistic mercury removal by conventional pollutant control strategies for coal-fired power plants in China, J. Air Waste Manag. Assoc., 6, 722-730, 2010b.

Wang, S. X., Song, J. X., Li, G. H., Wu, Y., Zhang, L., Wan, Q., Streets, D. G., Chin, C. K., Hao, J. M.: Estimating mercury emissions from a zinc smelter in relation to China's mercury control policies, Environ. Pollut., 158, 3347-3353, 2010c.

Wang, W., Qin, Y., Song, D.: Study on the mobility and release of trace elements in coal-fired power plant, Acta Scientiae Circumstantiae, 06, 748-752, 2003 (in Chinese).

Wang, X., Deng, S., Zhang, F., Wang, H., Liu, Y., Zhang, C., Cao, Q.: Coal fired power plant dust removal equipment synergy to take off the mercury research performance, the Fourteenth Annual Meeting of China Association for Science and Technology, Dust removal and desulfurization and mercury removal equipment, technology development and application seminar, 2012.

Wang, Y., Duan, Y., Yang, L., Jiang, Y.: Comparison of mercury removal characteristic between fabric filter and electrostatic precipitators of coal-fired power plants, Journal of Fuel Chemistry and Technology, 01, 23-29, 2008a(in Chinese).

Wang, Y., Duan Y., Yang, L., Meng, S., Wu, C., Wang, Q.: Experimental Study on mercury removal by combined wet flue gas desulphurization with electrostatic precipitator, Proceedings of the CSEE, 29, 64-69, 2008b (in Chinese).

Wang, Y., Duan, Y., Yang, L., Jiang, Y.: Analysis of the factors exercising an influence on the morphological transformation of mercury in the flue gas of a 600MW coal-fired power plant, Journal of Engineering for Thermal Energy and Power, 04, 399-403, 2008c (in Chinese).

Wang, Z.: Research on the laws of the mercury emissions of coal-fired power plants and the mechanism of cooperative control (Master Thesis), Nanjing Normal University, Nanjing, China, 2013 (in Chinese).

Wu, C., Cao, Y., Dong, Z., Cheng, C., Li, H., Pan, W.: Evaluation of mercury speciation and removal through air pollution control devices of a 190 MW boiler, Journal of Environmental Sciences, 02, 277-282, 2010.

Wu, Q. R., Wang, S. X., Zhang, L., Song, J. X., Yang, H., Meng, Y.: Update of mercury emissions from China's primary zinc, lead and copper smelters, 2000-2010. Atmos. Chem. Phys., 12, 11153-11163, 2012.

Wu, Y., Streets, D. G., Wang, S. X., Hao, J. M.: Uncertainties in estimating mercury emissions from coal-fired power plants in China, Atmos. Chem. Phys., 10, 2937-2947, 2010.

Wu, Y., Wang, S., Streets, D. G., Hao, J., Chan, M., Jiang. J.: Trends in anthropogenic mercury emissions in China from 1995 to 2003, Environ. Sci. Technol., 40, 5312-5318, 2006.

Xie, X., Yin, W.: Nanjing Thermal Power Plant Boiler Flue Gas Mercury Emissions in the Survey, Environmental Monitoring and Forewarning, 6, 47-49, 2014.

$\mathrm{Xu}$, W., Wang, J., Wang, W.: Effect of precipitator and desulphurization devices on the removal of mercury with different speciation in coal-fired flue gas, East China Electric Power, 01, 47-50, 2010 (in Chinese).

Yang, L., Duan, Y., Yang, X., Jiang, Y., Wang, Y., Zhao, C.: Mercury emission characteristic from 
coal-fired power plants, Journal of Southeast University (Natural Science Edition), 05, 817-821, 2007 (in Chinese).

Yang, X., Duan, Y., Jiang. Y., Yang, L.: Research on mercury form distribution in flue gas and fly ash of coal-fired boiler, Coal Science and Technology, 12, 55-58, 2007 (in Chinese).

Zhang, L.: Research on mercury emission measurement and estimate from combustion resources (Master Thesis), Zhejiang University, Hangzhou, China, 2007 (in Chinese).

Zhang, L.: Emission Characteristics and Synergistic Control Strategies of Atmospheric Mercury from Coal Combustion in China. Ph. D thesis, Tsinghua University, Beijing, China, 2012 (in Chinese).

Zhang, L., Wang, S., Wu, L., Wu, Y., Duan, L., Wu, Q., Wang, F., Yang, M., Yang, H., Hao, J., Liu, X.: Updated emission inventories for speciated atmospheric mercury from anthropogenic sources in china, Environ. Sci. Technol., 49, 3185-3194, 2015.

Zhang, L., Wang, S., Wu, Q., Meng, Y., Yang, H., Wang, F., Hao, J.: Were mercury emission factors for Chinese non-ferrous metal smelters overestimated? Evidence from onsite measurements in six smelters, Environ. Pollut., 171, 109-117, 2012.

Zhang, L., Zhuo, Y., Chen, L., Xu, X., Chen, C.: Mercury emissions from six coal-fired power plants in China, Fuel Processing Technology, 11, 1033-1040, 2008.

Zhang, W., Wei, W., Hu, D., Zhu, Y., Wang, X.: Emission of speciated mercury from residential biomass fuel combustion in China, Energy Fuels, 27, 6792-6800, 2013.

Zhao, Y., Zhong H., Zhang, J., Nielsen, P.: Evaluating the effects of China's pollution control on inter-annual trends and uncertainties of atmospheric mercury emissions, Atmos. Chem. Phys., 15, 4317-4337, 2015.

Zhi, G., Xue, Z., Li, Y., Ma, J., Liu, Y., Meng, F., Chai, F.: Uncertainty of flue gas mercury emissions from coal-fired power plants in China based on field measurements, Research of Environmental Sciences, 26, 814-821, 2013 (in Chinese).

Zhong, L., Cao, Y., Li, W., Pan, W., Xie, K.: Effect of the existing air pollutant control devices on mercury emission in coal-fired power plants, Journal of Fuel Chemistry and Technology, 6, 641-646, 2010 (in Chinese).

Zhou, J., Zhang, L., Luo, Z., Hu. C., He, S., Zheng, J., Cen, K.: Research progress of removing mercury from coal-fired flue gas, Chemical Industry and Engineering Progress, 04, 22-27, 2008 (in Chinese).

Zhu, J, Tan, Y., Zheng, J., Zhang, C., Li, Y., Zhang, D., Wang, C.: Study on characteristic of mercury distribution in combustion products at various loads of a P.C.-fired utility boiler, Proceedings of the CSEE, 07, 87-90, 2001 (in Chinese). 